\title{
18 Cholesterol and arteriosclerosis
}

The endothelium, the layer of cells lining the interior of blood vessels, forms a complicated interface that uses signals carried by the circulating blood to modulate vascular tone, the concentration of inflammatory cells and the coagulation cascade. Factors causing the endothelium to malfunction are high levels of LDL cholesterol, which in turn increase the associated risk for atherosclerosis. When macrophages attempt to remove this LDL cholesterol from the vascular lesions by oxidative digestion, highly reactive oxygen compounds are continuously released. These high-energy radicals inactivate the nitric oxide produced by the endothelium and important for normal vascular function ( $\triangleright$ Chapter 51). A further classification of LDL particle diameters by size reveals that it is mostly the very small and very large particles that pose the greatest risk (Grammer et al. 2014).

The molecules of the HDL fraction, likewise consisting of many subgroups, transport building blocks for the synthesis of nitric oxide along with messenger substances that reduce inflammatory reactions. Among others, one very important task of these HDL particles also consists of removing harmful cholesterol from the circulation in a process called reverse cholesterol transport to the liver ( $\triangleright$ Chapter 17). During this process, they are able to accept cholesterol breakdown products from the macrophages working in the arterial wall. The more effective this efflux process runs, the lower is the probability of coronary artery disease, independently of the level of HDL cholesterol (Khera et al. 2011).

Too high levels of total cholesterol require treatment. However, this no longer applies globally to only moderately elevated levels, as these are not generally a health risk. In 2013, this finding gave reason to correct the previous recommendation by cardiologists that cholesterol levels elevated above $200 \mathrm{mg} / \mathrm{dL}$ should strictly be regarded as requiring treatment (Stone et al. 2014, Lloyd-Jones et al. 2014). Now, cholesterol-lowering interventions are only indicated in patients with cardiovascular disease and diabetes or in individuals with a statistically elevated risk for myocardial infarction or stroke as well as for those with markedly elevated LDL cholesterol levels $>190 \mathrm{mg} / \mathrm{dL}$.

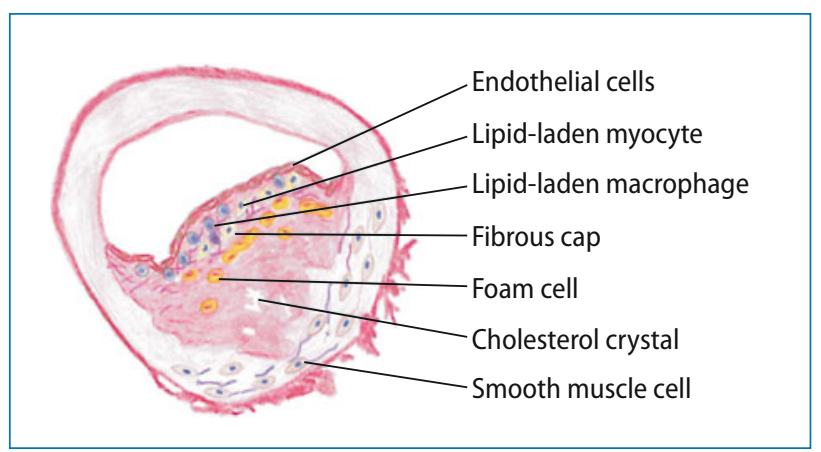

Fig. 18.1 Vasoconstriction in arteriosclerosis 